Received by the editors: June 13, 2020; Accepted: July 06, 2020

\title{
PRODUCT FACTORABLE MULTILINEAR OPERATORS DEFINED ON SEQUENCE SPACES
}

\author{
Ezgi ERDOĞAN \\ Marmara University, Faculty of Arts and Sciences, Department of Mathematics, TR-34722, \\ Kadıköy, Istanbul, TURKEY
}

\begin{abstract}
We prove a factorization theorem for multilinear operators acting in topological products of spaces of (scalar) $p$-summable sequences through a product. It is shown that this class of multilinear operators called product factorable maps coincides with the well-known class of the zero product preserving operators. Due to the factorization, we obtain compactness and summability properties by using classical functional analysis tools. Besides, we give some isomorphisms between spaces of linear and multilinear operators, and representations of some classes of multilinear maps as $n$-homogeneous orthogonally additive polynomials.
\end{abstract}

\section{INTRODUCTION}

The objective of the paper is to present a factorization theorem for multilinear operators defined on the topological product of spaces of $p$-summable sequences through the product of (multiple) scalar sequences. Such a factorization has been studied for multilinear operators defined on Banach algebras and vector lattices, and in the last years it has been studied for Banach spaces (see 1, 6, 12 and references therein).

Factorization through a product is closely related to a property that is called zero product preservation, or orthosymmetry in the case of vector lattices, for which orthogonality is used to generalize the notion of having product equal to 0 , that is just given for the case of function lattices. This property states that a multilinear map $B: X_{1} \times \ldots \times X_{n} \rightarrow Y$ is 0 -valued whenever $x_{i} \circledast x_{j}=0$ for some $x_{i} \in X_{i}$, $x_{j} \in X_{j}(i, j \in\{1,2, \ldots, n\})$, where $\circledast: X_{1} \times \ldots \times X_{n} \rightarrow G$ is a specific map

2020 Mathematics Subject Classification. Primary 47H60, 47A68; Secondary 46B45, 46B42.

Keywords and phrases. Sequence spaces, multilinear operators, factorization, zero product preserving map, polynomials.

घezgi.erdogan@marmara.edu.tr

(D) 0000-0002-0641-1930

(C)2020 Ankara University Communications Faculty of Sciences University of Ankara-Series A1 Mathematics and Statistics 
called product. For multilinear operators acting in Banach algebras, this factorization gives useful results for the weighted homomorphisms and derivations, where algebraic multiplication is considered as the specific map (see 1, 1,2 and references therein). For Riesz spaces, such a factorization is used to obtain interesting results regarding powers of vector lattices, in which orthogonality is involved (see [4, 6, 7 and references therein).

Recently, the author together with other mathematicians have investigated the class of multilinear operators acting in the topological product of Banach function spaces and integrable functions factoring through the pointwise product and the convolution operation, respectively (see [12 14]). Motivated by these ideas, in this paper we introduce the notion of product factorability for multilinear operators defined on topological products of spaces of (scalar) $p$-summable sequences, and we prove that this class coincides with the class of zero product preserving multilinear maps.

This paper is organised as follows: after some preliminaries and notations, in Section 2 we give the definitions of the specific map product and product factorability for multilinear operators with a necessary and sufficient requirement. Section 3 includes the main result of the paper, which as we said above, states that for a particular product and multilinear operators defined on the topological product of spaces of $p$-summable sequences, the class of product factorable maps is the same as the class of zero product preserving maps. In the sequel, some isometries between multilinear operators and linear operators are presented. Section 4 concerns compactness and summability properties based on classical functional analysis properties and theorems of product factorable maps. Section 5 is devoted to give a generalization of the main factorization theorem by using isomorphism between Banach spaces and $\ell^{p}$ spaces. In the last section, some isometries between product factorable multilinear maps and orthogonally additive $n$-homogeneous polynomials are given as an application, and the paper is finished with an example related to diagonal forms.

Throughout the paper, the standard notations from the Banach space theory are used. Nevertheless, before going any further let us describe some of them. The capital letters $X, Y, Z$ will denote the Banach spaces over the scalar field $\mathbb{K}=\mathbb{R}$ or $\mathbb{C}$. We write $B_{X}$ for the unit ball of a Banach space $X$. $X^{*}$ denotes the topological dual of the Banach space $X$. The notations $E=Y$ and $E \cong Y$ mean $E$ and $Y$ are isometric and isomorphic, respectively.

Operator (linear, multilinear or polynomial) indicates continuous operator. $\mathcal{L}^{n}\left(X_{1} \times \ldots \times X_{n}, Y\right)$ denotes the Banach space of $n$-linear maps endowed with the norm

$$
\|T\|=\sup \left\{\left\|T\left(x_{1}, \ldots, x_{n}\right)\right\|: x_{i} \in B_{X_{i}}, 1 \leq i \leq n\right\} .
$$

It will be denoted by $\mathcal{L}^{n}\left(X_{1} \times \ldots \times X_{n}\right)$, respectively, $\mathcal{L}(X, Y)$ if $Y=\mathbb{R}$, respectively, $n=1$. 
For a positive real number $p \geq 1, \ell^{p}$ is the Banach space of all scalar valued absolutely $p$-summable sequences with the norm $\left\|\left(x_{i}\right)\right\|_{p}=\left(\sum_{i=1}^{\infty}\left|x_{i}\right|^{p}\right)^{1 / p}$ and $\ell^{\infty}$ shows the Banach space of all bounded sequences endowed with the norm $\left\|\left(x_{i}\right)\right\|_{\infty}=$ $\sup _{i \in \mathbb{N}}\left|x_{i}\right|$.

$\chi_{\{1,2, \ldots, m\}}$ will denote the sequence $\{1, m 1,0,0,0 \ldots$.$\} and \chi_{\{j\}}$ shows the elements of standard basis of the space $\ell^{p}$ whose coordinates are all zero, except $j^{\text {th }}$ that equals 1 .

For brevity we will write $\times_{i=1}^{n} X_{i}$ for the Cartesian product space $X_{1} \times \ldots \times X_{n}$ and $\times^{n} X$ for the $n$-fold Cartesian product of the Banach space $X$.

A linear operator $T: X \rightarrow Y$ is called $(p, q)$-summing if there exists a constant $c>0$ such that for every choice of the elements $x_{1}, \ldots, x_{m} \in X$ and for all positive integers $m$,

$$
\left(\sum_{i=1}^{m}\left\|T\left(x_{i}\right)\right\|_{Y}^{p}\right)^{1 / p} \leq k \sup _{x^{*} \in B_{X^{*}}}\left(\sum_{i=1}^{m}\left|\left\langle x_{i}, x^{*}\right\rangle\right|^{q}\right)^{1 / q} .
$$

The space of $(p, q)$-summing operators from $X$ to $Y$ is denoted by $\Pi_{p, q}(X, Y)-$ $-\Pi_{p}(X, Y)$, if $p=q$.

Recall that a Banach space $E$ is said to have the Schur property whenever weak convergent and norm convergent sequences coincide in it. A Banach space $E$ has the Dunford-Pettis property if every linear operator from $E$ into a Banach space $F$ maps weakly compact sets to norm compact ones.

Recall that an (linear, multilinear or polynomial) operator is called (weakly) compact if it maps the unit ball to a relatively (weakly) compact set.

\section{Norm Preserving Products and Product Factorability}

Let $X_{1}, X_{2}, \ldots, X_{n}$ and $Z$ be Banach spaces. Consider a Banach space valued $n$-linear map $\circledast: X_{1} \times X_{2} \times \ldots \times X_{n} \rightarrow Z$ written by

$$
\left(x_{1}, x_{2}, \ldots, x_{n}\right) \rightsquigarrow \circledast\left(x_{1}, x_{2}, \ldots, x_{n}\right)=x_{1} \circledast x_{2} \circledast \ldots \circledast x_{n}
$$

for all $x_{i} \in X_{i}(i=1,2, \ldots, n)$.

This particular map is called norm preserving product (n.p. product for short) if the inclusion $B_{Y} \subseteq \circledast\left(B_{X_{1}} \times B_{X_{2}} \times \ldots \times B_{X_{n}}\right)$ holds and for every $x_{i} \in X_{i}(i=$ $1, \ldots, n)$ and we have that

$$
\left\|\circledast\left(x_{1}, x_{2}, \ldots, x_{n}\right)\right\|_{Z}=\inf \left\{\prod_{i=1}^{n}\left\|x_{i}^{\prime}\right\|_{X_{i}}: x_{i}^{\prime} \in X_{i}, i=1, \ldots, n\right\},
$$

where the infimum is taken over all $\circledast\left(x_{1}, x_{2}, \ldots, x_{n}\right)=\circledast\left(x_{1}^{\prime}, x_{2}^{\prime}, \ldots, x_{n}^{\prime}\right)$ (see 12 , Definition 2.1]) .

Example 1. Some norm preserving products; 
- The usual convolution operation $*$ from the product $\mathcal{L}^{2}(\mathbb{T}) \times \mathcal{L}^{2}(\mathbb{T})$ of Hilbert space of integrable functions to the Wiener algebra $\mathcal{W}(\mathbb{T})$ is a norm preserving product (see [11, Remark 2.1] and references there in for the calculations),

- Let $(\Omega, \Sigma, \mu)$ be a $\sigma$-finite measure space and let $\sum_{i=1}^{n} \frac{1}{p_{i}}=\frac{1}{r}$ and $p_{i}, r \geq 1$. Then the pointwise product $\odot$ defined on $\mathcal{L}^{p_{1}}(\mu) \times \ldots \times \mathcal{L}^{p_{n}}(\mu)$ to $\mathcal{L}^{r}(\mu)$ is a norm preserving product (see [12, Section 4]).

A multilinear operator $B: X_{1} \times \ldots \times X_{n} \rightarrow Y$ is called $\circledast$-factorable for the n.p. product $\circledast$ if it can be factored through the product $\circledast: X_{1} \times \ldots \times X_{n} \rightarrow Z$ and a linear operator $T: Z \rightarrow Y$ such that $B\left(x_{1}, x_{2}, \ldots, x_{n}\right)=T \circ \circledast\left(x_{1}, x_{2}, \ldots, x_{n}\right)=$ $T\left(x_{1} \circledast x_{2} \circledast \ldots \circledast x_{n}\right)$ for all $x_{i} \in X_{i}(i=1, \ldots, n)$ (see [12, Def. 2.2]).

Thus, for a certain continuous linear operator $T: Z \rightarrow Y$, the map $B$ admits a factorization as the form;

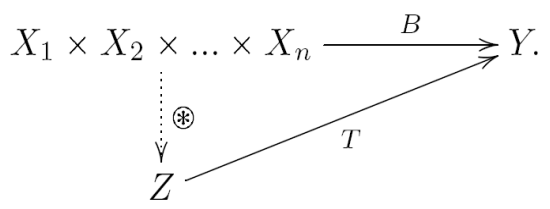

The author proved in [12, Lemma 2.3.] that a necessary and sufficient condition for the $\circledast$-factorability of a multilinear operator $B: X_{1} \times X_{2} \times \ldots \times X_{n} \rightarrow Y$ is given by the existence of a constant $k>0$ satisfying the following inequality

$$
\left\|\sum_{i=1}^{m} B\left(x_{i}^{1}, x_{i}^{2}, \ldots, x_{i}^{n}\right)\right\|_{Y} \leq k\left\|\sum_{i=1}^{m} x_{i}^{1} \circledast x_{i}^{2} \circledast \ldots \circledast x_{i}^{n}\right\|_{Z}
$$

for every finite sets of vectors $\left\{x_{i}^{j}\right\}_{i=1}^{m} \subset X_{j}(j=1,2, \ldots, n)$.

A multilinear map $B: X_{1} \times X_{2} \times \ldots \times X_{n} \rightarrow Y$ is called zero product preserving (or zero $\circledast$-preserving) if

$$
B\left(x_{1}, x_{2}, \ldots, x_{n}\right)=0 \text { if } x_{k} \circledast x_{l}=0 \text { for some } x_{k} \in X_{k}, x_{l} \in X_{l}
$$

where $k, l \in\{1,2, \ldots, n\}$ and $k \neq l$.

The class of zero $\circledast$-preserving multilinear operators is a Banach space endowed with the usual operator norm. The Banach space of $n$-linear zero $\circledast$-preserving operators defined on the $X_{1} \times X_{2} \times \ldots \times X_{n}$ to $Y$ will be denoted by $\mathcal{L}_{0}^{n}\left(X_{1} \times X_{2} \times\right.$ $\left.\ldots \times X_{n}, Y\right)$.

\section{Product Factorability of Multilinear Maps acting in Sequence SPACES}

Now, we will give the main theorem of the paper that states the class of zero product preserving maps defined on $\times_{i=1}^{n} \ell^{p_{i}}$ to the Banach space $Y$ are equal to the class of the product factorable operators. 
Remark 2. Let $\sum_{i=1}^{n} \frac{1}{p_{i}}=\frac{1}{r}$ for $1 \leq r, p_{i}<\infty(i=1, \ldots, n)$. The product $\odot: \times_{i=1}^{n} \ell^{p_{i}} \rightarrow \ell^{r}$ defined by

$$
x_{1} \odot \ldots \odot x_{n}=\left\{x^{1}(k) \cdot \ldots . x^{n}(k)\right\}_{k=1}^{\infty}=\{x(k)\}_{k=1}^{\infty}=x \in \ell^{r}
$$

for all $x_{i} \in \ell^{p_{i}}(i=1, \ldots, n)$ is a norm preserving product. Indeed, consider a $\{x(k)\}_{k=1}^{\infty} \in B_{\ell^{r}}$. We can write $x(k)=\prod_{i=1}^{n}|x(k)|^{r / p_{i}} \operatorname{sign}(x(k))$ for all $k \in \mathbb{N}$, where sign denotes the signum function. Since

$$
\begin{aligned}
\left\|\left(|x(k)|^{r / p_{i}} \operatorname{sign}(x(k))\right)\right\|_{p_{i}} & =\left(\sum \|\left.\left. x(k)\right|^{r / p_{i}} \operatorname{sign}(x(k))\right|^{p_{i}}\right)^{1 / p_{i}} \\
& =\left(\sum|x(k)|^{r}\right)^{1 / p_{i}}=\|(x(k))\|_{r}^{r / p_{i}} \leq 1,
\end{aligned}
$$

we get $\left\{|x(k)|^{r / p_{i}} \operatorname{sign}(x(k))\right\}_{k=1}^{\infty} \in \ell^{p_{i}}$ and $B_{\ell^{r}} \subseteq \odot\left(B_{\ell^{p_{1}}} \times B_{\ell^{p_{2}}} \times \ldots \times B_{\ell^{p_{n}}}\right)$. Now, let us show the equality given in the definition of the n.p. product. Take into account sequences $x_{i}=\left\{x^{i}(k)\right\}_{k=1}^{\infty} \in \ell^{p_{i}}$ for $i=1,2, \ldots, n$ such that $x_{1} \odot x_{2} \odot \ldots \odot x_{n}=x$.

By the generalization of Hölder's inequlity it is easily seen that

$$
\left\|x_{1} \odot x_{2} \odot \ldots \odot x_{n}\right\|_{r} \leq\left\|x_{1}\right\|_{p_{1}}\left\|x_{2}\right\|_{p_{2} \ldots} \ldots x_{n} \|_{p_{n}} .
$$

Now, let us show the inverse. Since for all $k$, we can write $x(k)=\prod_{i=1}^{n}|x(k)|^{r / p_{i}} \operatorname{sign}(x(k))$, we get $\left\|\left(|x(k)|^{r / p_{i}} \operatorname{sign}(x(k))\right)\right\|_{p_{i}}=\|(x(k))\|_{r}^{r / p_{i}}$. Therefore

$$
\|x\|_{r}=\|(x(k))\|_{r}=\prod_{i=1}^{n}\|(x(k))\|_{r}^{r / p_{i}}=\prod_{i=1}^{n}\left\|\left(|x(k)|^{r / p_{i}} \operatorname{sign}(x(k))\right)\right\|_{p_{i}} .
$$

Thus, we get $\|x\|_{r}=\inf \left\{\left\|x_{1}\right\|_{p_{1}}\left\|x_{2}\right\|_{p_{2}} \ldots\left\|x_{n}\right\|_{p_{n}}\right\}$ and $\odot$ is an n.p. product from $\ell^{p_{1}} \times \ell^{p_{2}} \times \ldots \times \ell^{p_{n}}$ to $\ell^{r}$.

Theorem 3. Let $\sum_{i=1}^{n} \frac{1}{p_{i}}=\frac{1}{r}$ for $1 \leq r, p_{i}<\infty(i=1, \ldots, n)$. For a multilinear operator $B: \times_{i=1}^{n} \ell^{p_{i}} \rightarrow Y$ the following statements imply each other.

(1) The operator $B$ is zero $\odot$-preserving.

(2) The operator $B$ is $\odot$-factorable.

(3) There is a constant $k>0$ such that for every finite sets of sequences $\left\{x_{1}^{i}, \ldots, x_{m}^{i}\right\} \subset \ell^{p_{i}}(i=1,2, \ldots, n)$, the following inequality holds;

$$
\left\|\sum_{j=1}^{m} B\left(x_{j}^{1}, x_{j}^{2}, \ldots, x_{j}^{n}\right)\right\|_{Y} \leq k\left\|\sum_{j=1}^{m} x_{j}^{1} \odot x_{j}^{2} \odot \ldots \odot x_{j}^{n}\right\|_{r} .
$$

Thus, $B$ admits the following factorization for a unique linear operator $T: \ell^{r} \rightarrow Y$;

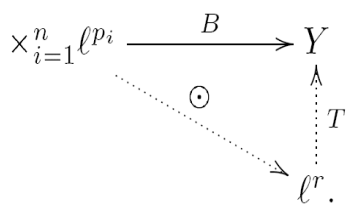


Proof. (1) $\Rightarrow(2)$ Assume that $B$ is zero $\odot$-preserving. Let us write the sequences $x_{i} \in \ell^{p_{i}}(i=1,2, \ldots, n)$ in the form $x_{i}=\left\{x^{i}(k)\right\}_{k=1}^{\infty}=\sum_{k=1}^{\infty} x^{i}(k) \chi_{\{k\}}$, then

$$
x_{1} \odot \ldots . \odot x_{n}=\left\{x^{1}(k) \cdot \ldots . \cdot x^{n}(k)\right\}_{k=1}^{\infty}=\sum_{k=1}^{\infty} x^{1}(k) \cdot \ldots . x^{n}(k) \cdot \chi_{\{k\}}
$$

Since $\chi_{\{k\}} \odot \chi_{\{l\}}=0$ whenever $k \neq l$, the following equality is obtained

$$
\begin{aligned}
B\left(x_{1}, \ldots, x_{n}\right) & =B\left(\sum_{k_{1}=1}^{\infty} x^{1}\left(k_{1}\right) \chi_{\left\{k_{1}\right\}}, \ldots, \sum_{k_{n}=1}^{\infty} x^{n}\left(k_{n}\right) \chi_{\left\{k_{n}\right\}}\right) \\
& =\sum_{k_{1}=1}^{\infty} x^{1}\left(k_{1}\right) \cdot \ldots \cdot \sum_{k_{n}=1}^{\infty} x^{n}\left(k_{1}\right) B\left(\chi_{\left\{k_{1}\right\}}, \ldots, \chi_{\left\{k_{n}\right\}}\right) \\
& =\sum_{k=1}^{\infty} x^{1}(k) \cdot \ldots \cdot x^{n}(k) B\left(\chi_{\{k\}}, \ldots, \chi_{\{k\}}\right) \\
& =B\left(\sum_{k=1}^{\infty} x^{1}(k) \cdot \ldots \cdot x^{n}(k) \chi_{\{k\}}, \chi_{\{k\}}, \ldots, \chi_{\{k\}}\right)
\end{aligned}
$$

by the zero product preservartion property of $B$.

For every natural number $m$, let us define the map $B_{m}\left(x_{1}, \ldots, x_{n}\right)=B\left(x_{1} \odot\right.$ $\left.\chi_{\{1, \ldots, m\}}, \ldots, x_{n} \odot \chi_{\{1, \ldots, m\}}\right)$ for all $x_{i} \in \ell^{p_{i}}(i=1,2, \ldots, n)$. It is easily seen that the sequence $\left\{B_{m}\right\}_{m=1}^{\infty}$ consists of well-defined, multilinear continuous maps. Since $x_{i} \odot \chi_{\{1, \ldots, m\}}=x_{i} \odot \sum_{k=1}^{m} \chi_{\{k\}}=\sum_{k=1}^{m} x^{i}(k) \chi_{\{k\}}$, by the zero $\odot$-preservation property of $B$

$$
\begin{aligned}
B_{m}\left(x_{1}, \ldots, x_{n}\right) & =B\left(x_{1} \odot \chi_{\{1, \ldots, m\}}, \ldots, x_{n} \odot \chi_{\{1, \ldots, m\}}\right) \\
& =B\left(\sum_{k_{1}=1}^{m} x^{1}\left(k_{1}\right) \chi_{\left\{k_{1}\right\}}, \ldots, \sum_{k_{n}=1}^{m} x^{n}\left(k_{n}\right) \chi_{\left\{k_{n}\right\}}\right) \\
& =\sum_{k_{1}=1}^{m} x^{1}\left(k_{1}\right) \cdot \ldots \cdot \sum_{k_{n}=1}^{m} x^{n}\left(k_{n}\right) B\left(\chi_{\left\{k_{1}\right\}}, \ldots, \chi_{\left\{k_{n}\right\}}\right) \\
& =\sum_{k=1}^{m} x^{1}(k) \cdot \ldots \cdot x^{n}(k) B\left(\chi_{\{k\}}, \ldots, \chi_{\{k\}}\right) \\
& =B\left(\sum_{k=1}^{m} x^{1}(k) \cdot \ldots \cdot x^{n}(k) \chi_{\{k\}}, \sum_{k=1}^{m} \chi_{\{k\}}, \ldots, \sum_{k=1}^{m} \chi_{\{k\}}\right) .
\end{aligned}
$$

Thus, for all $m$, the map $B_{m}$ is written as

$$
\begin{aligned}
B_{m}\left(x_{1}, \ldots, x_{n}\right) & =B\left(\sum_{j=1}^{m} x_{j}^{1} \cdot \ldots \cdot x_{j}^{n} \chi_{\{j\}}, \sum_{j=1}^{m} \chi_{\{j\}}, \ldots, \sum_{j=1}^{m} \chi_{\{j\}}\right) \\
& =B\left(x_{1} \odot \ldots \odot x_{n} \odot \chi_{\{1, \ldots, m\}}, \chi_{\{1, \ldots, m\}}, \ldots, \chi_{\{1, \ldots, m\}}\right)
\end{aligned}
$$


for all $x_{i} \in \ell^{p_{i}}(i=1,2, \ldots, n)$.

Now, for all natural number $m$ and every $x=x_{1} \odot \ldots \odot x_{n}$, define the map $T_{m}: \ell^{r} \rightarrow Y$ by $T_{m}(x)=T_{m}\left(x_{1} \odot \ldots \odot x_{n}\right)=B_{m}\left(x_{1}, \ldots, x_{n}\right)$. Then, it is seen that for all $m$, the map $T_{m}$ is well-defined, linear and continuous operator. Indeed, the linearity is seen by the linearity in the first variable of the map $B_{m}$. Let us show the continuity of the map $T_{m}$;

$$
\begin{aligned}
\left\|T_{m}(x)\right\|_{Y} & =\left\|B_{m}\left(x_{1}, \ldots, x_{n}\right)\right\|_{Y} \\
& =\left\|B\left(x_{1} \odot \chi_{\{1, \ldots, m\}}, \ldots, x_{n} \odot \chi_{\{1, \ldots, m\}}\right)\right\|_{Y} \\
& \leq\|B\|\left\|x_{1} \odot \chi_{\{1, \ldots, m\}}\right\| \ldots\left\|x_{n} \odot \chi_{\{1, \ldots, m\}}\right\| \\
& \leq\|B\|\left\|x_{1}\right\| \ldots\left\|x_{n}\right\|,
\end{aligned}
$$

since this holds for all representations of the sequence $x$, it is seen that $\left\|T_{m}(x)\right\|_{Y} \leq$ $\|B\|\|x\|_{r}$ by the definition of n.p. product. For all $m$, the operator $T_{m}$ is independent of the representation of the sequence $x$. Indeed, let us assume $x=x_{1} \odot \ldots \odot x_{n}=$ $x_{1}^{\prime} \odot \ldots \odot x_{n}^{\prime}$, then it is seen that

$$
\begin{aligned}
T_{m}\left(x_{1} \odot \ldots \odot x_{n}\right) & =B\left(x_{1} \odot \ldots \odot x_{n} \odot \chi_{\{1, \ldots, m\}}, \chi_{\{1, \ldots, m\}}, \ldots, \chi_{\{1, \ldots, m\}}\right) \\
& =B\left(x_{1}^{\prime} \odot \ldots \odot x_{n}^{\prime} \odot \chi_{\{1, \ldots, m\}}, \chi_{\{1, \ldots, m\}}, \ldots, \chi_{\{1, \ldots, m\}}\right) \\
& =T_{m}\left(x_{1}^{\prime} \odot \ldots \odot x_{n}^{\prime}\right) .
\end{aligned}
$$

On the other hand, the set of operators $\left\{T_{m}\right\}_{m=1}^{\infty}$ is pointwise convergent for each $x=x_{1} \odot \ldots \odot x_{n} \in \ell^{r}$. By the separate continuity of the multilinear map $B$, this is seen as follows;

$$
\begin{aligned}
\lim _{m \rightarrow \infty} T_{m}\left(x_{1} \odot \ldots \odot x_{n}\right) & =\lim _{m \rightarrow \infty} B_{m}\left(x_{1}, \ldots, x_{n}\right) \\
& =\lim _{m \rightarrow \infty} B\left(x_{1} \odot \chi_{\{1, \ldots, m\}}, \ldots, x_{n} \odot \chi_{\{1, \ldots, m\}}\right) \\
& =B\left(\lim _{m \rightarrow \infty} x_{1} \odot \chi_{\{1, \ldots, m\}}, \ldots, \lim _{m \rightarrow \infty} x_{n} \odot \chi_{\{1, \ldots, m\}}\right) \\
& =B\left(x_{1}, \ldots, x_{n}\right) .
\end{aligned}
$$

Thus, $\left\{T_{m}(x)\right\}_{m=1}^{\infty}$ converges to $B\left(x_{1}, \ldots, x_{n}\right)$ for all $x \in \ell^{r}$ such that $x=x_{1} \odot \ldots \odot x_{n}$ for the elements $x_{i} \in \ell^{p_{i}}(i=1, \ldots, n)$. Let us define the pointwise limit $T(x)=$ $\lim _{m \rightarrow \infty} T_{m}(x)$. It is clear that the limit map $T$ is well-defined and linear. Besides it is continuous by the uniform boundedness theorem.

Summing up, the linear bounded map $T: \ell^{r} \rightarrow Y$ defined by $T\left(x_{1} \odot \ldots \odot x_{n}\right)=$ $B\left(x_{1}, \ldots, x_{n}\right)$ is the desired map.

$(2) \Rightarrow(3)$ is obtained by Lemma 2.3. given in 12 .

Lastly, let us show (3) implies (1). Consider the sequences $x_{i} \in \ell^{p_{i}}(i=1, \ldots, n)$ such that $x_{k} \odot x_{l}=0$ for some different $k, l \in\{1, \ldots, n\}$. This implies $x_{1} \odot \ldots \odot x_{n}=0$. Therefore, zero $\odot$-preservation is seen by Inequality $(2)$ given in the statement (3). 
The above theorem gives an isometry between the spaces $\mathcal{L}_{0}^{n}\left(\times_{i=1}^{n} \ell^{p_{i}}, Y\right)$ and $\mathcal{L}\left(\ell^{r}, Y\right)$.

Theorem 4. The correspondence $B \longleftrightarrow T$ is an onto isometry between the Banach spaces $\mathcal{L}_{0}^{n}\left(\times_{i=1}^{n} \ell^{p_{i}}, Y\right)$ and $\mathcal{L}\left(\ell^{r}, Y\right)$.

Particularly for $Y=\mathbb{R}$, we get $\mathcal{L}_{0}^{n}\left(\times_{i=1}^{n} \ell^{p_{i}}\right)=\left(\ell^{r}\right)^{*}$.

Proof. It is easily seen that the map $\mathcal{L}_{0}^{n}\left(\times_{i=1}^{n} \ell^{p_{i}}, Y\right) \rightarrow \mathcal{L}\left(\ell^{r}, Y\right)$ is linear. Now, let us show the isometry.

$$
\begin{aligned}
\|B\| & =\sup _{\left(x_{1}, \ldots, x_{n}\right) \in \times_{i=1}^{n} B_{\ell} p_{i}}\left\|B\left(x_{1}, \ldots, x_{n}\right)\right\|_{Y} \\
& =\sup _{\left(x_{1}, \ldots, x_{n}\right) \in \times_{i=1}^{n} B_{\ell^{p_{i}}}}\left\|T\left(x_{1} \odot \ldots \odot x_{n}\right)\right\|_{Y} \\
& \geq \sup _{x=x_{1} \odot \ldots \odot x_{n} \in B_{\ell^{r}}}\|T x\|_{Y}=\|T\| .
\end{aligned}
$$

For the converse inequality;

$$
\|T\|=\sup _{x \in B_{\ell^{r}}}\|T x\|_{Y}=\sup _{\left(x_{1}, \ldots, x_{n}\right) \in \times_{i=1}^{n} B_{\ell^{p_{i}}}}\left\|B\left(x_{1}, \ldots, x_{n}\right)\right\| \leq\|B\|,
$$

where $x_{i}=\left\{x^{i}(k)\right\}_{k=1}^{\infty}=\left\{|x(k)|^{r / p_{i}} \operatorname{sgn}(x(k))\right\}_{k=1}^{\infty}$ for all $i=1, \ldots, n$.

It is easily seen that the map $B \rightarrow T$ is onto, since an $n$-linear map $B_{T}$ is obtained for every linear map $T$ by defining $T(x)=B\left(x_{1}, \ldots, x_{n}\right)$ for all $x=x_{1} \odot \ldots \odot x_{n} \in \ell^{r}$ for the n.p product $\odot: \times_{i=1}^{n} \ell^{p_{i}} \rightarrow \ell^{r}$.

Corollary 5. As a result of the above isometry, the following isometries are given for particular $p_{i}$ values.

$\star \mathcal{L}_{0}^{n}\left(\times^{n} \ell^{p}, Y\right)=\mathcal{L}\left(\ell^{p / n}, Y\right)$, where $p>n$.

$\star \mathcal{L}_{0}^{n}\left(\times^{n} \ell^{n}, Y\right)=\mathcal{L}\left(\ell^{1}, Y\right)$

$\star \mathcal{L}_{0}^{n}\left(x^{n} \ell^{p}\right)=\left(\ell^{p / n}\right)^{*}=\ell^{p /(p-n)}$

$\star \mathcal{L}_{0}^{n}\left(\times^{n} \ell^{n}\right)=\left(\ell^{1}\right)^{*}=\ell^{\infty}$.

\section{Compactness and Summability Inquiries for $\odot$-Factorable Maps}

In this section, we investigate compactness and summability for $\odot$-factorable multilinear operator that are based on the classical analysis properties and theorems like Dunford Pettis property, well-known Grothendieck's theorem or cotype related properties.

4.1. Compactness of $\odot$-Factorable operators. By the definition of norm preserving product, it is seen that a $\odot$-factorable multilinear map $B: \times_{i=1}^{n} \ell^{p_{i}} \rightarrow Y$ is (weakly) compact if and only if the linear operator $T: \ell^{r} \rightarrow Y$ appearing in the factorization is (weakly) compact. Now, we will give more specific compactness implications for $\odot$-factorable maps. 
Corollary 6. Let $\sum_{i=1}^{n} \frac{1}{p_{i}}=\frac{1}{r}$ for $1 \leq r, p_{i}<\infty$ and $i=1, \ldots, n$. For $a \odot-$ factorable multilinear operator $B: \times_{i=1}^{n} \ell^{p_{i}} \rightarrow Y$, we have the following compactness results;

(1) For $r>1$, the map $B$ is weakly compact.

(2) If $r=1$ and $Y$ is reflexive, then the map $B$ is compact.

(3) For $1 \leq s<r<\infty$ and $Y=\ell^{s}$, the map $B$ is compact.

Proof. (1) This is easily seen by the weakly compactness of the factorization operator $T: \ell^{r} \rightarrow Y$ which is defined on the reflexive space $\ell^{r}$.

(2) $B$ factors through the linear map $T: \ell^{r} \rightarrow Y$ that is weakly compact due to reflexivity of the space $Y$. In addition, $T$-hence $B$ - is compact by the DunfordPettis property of the space $\ell^{1}$.

(3) Since the linear operator $T: \ell^{r} \rightarrow \ell^{s}$ is compact whenever $1 \leq s<r<\infty$ by the Pitt's theorem, the map $B$ is so also (see $[9$, Chapter 12]).

Corollary 7. Let $\sum_{i=1}^{n} \frac{1}{p_{i}}=1$ for $1 \leq p_{i}<\infty(i=1, \ldots, n)$ and let $B: \times_{i=1}^{n} \ell^{p_{i}} \rightarrow$ $Y$ be $a \odot$-factorable multilinear operator. For a set $A \subset \times_{i=1}^{n} \ell^{p_{i}}, B(A)$ is norm compact if $\left\{x_{1} \odot \ldots \odot x_{n}:\left(x_{1}, \ldots, x_{n}\right) \in \ell^{p_{1}} \times \ldots \times \ell^{p_{n}}\right\}$ is weakly compact.

Proof. The $\odot$-factorable multilinear operator $B$ factors throug a linear map $T$ : $\ell^{1} \rightarrow Y$. Since $\odot(A)$ is weakly compact, $B(A)=T \circ \odot(A)$ is weakly compact. Hence it is compact by the Dunford-Pettis property of $\ell^{1}$.

4.2. Summability Properties of $\odot$-Factorable Operators. Now, let us look at the summability properties of $\odot$-factorable maps.

Theorem 8. Let $\sum_{i=1}^{n} \frac{1}{p_{i}}=1$ for $1 \leq p_{i}<\infty(i=1, \ldots, n)$. The followings imply each other for a Hilbert-space valued multilinear map $B: \times_{i=1}^{n} \ell^{p_{i}} \rightarrow H$.

i) The map $B$ is $\odot$-factorable,

ii) There is a constant $k>0$ such that for every finite sets $\left\{x_{1}^{i}, \ldots, x_{m}^{i}\right\} \subset$ $\ell^{p_{i}}(i=1, \ldots, n)$

$$
\sum_{j=1}^{m}\left\|B\left(x_{j}^{1}, \ldots, x_{j}^{n}\right)\right\|_{H} \leq k \sup _{z^{\prime} \in B_{\ell} \infty} \sum_{j=1}^{m}\left|\left\langle x_{j}^{1} \odot \ldots \odot x_{j}^{n}, z^{\prime}\right\rangle\right|,
$$

iii) For all $x_{i} \in \ell^{p_{i}}(i=1, \ldots, n)$ there is a regular Borel measure $\eta$ over $B_{\ell^{\infty}}$ such that

$$
\left\|B\left(x_{1}, \ldots, x_{n}\right)\right\|_{H} \leq K \int_{B_{\ell} \infty}\left|\left\langle x_{1} \odot \ldots \odot x_{n}, z^{\prime}\right\rangle\right| d \eta\left(z^{\prime}\right) .
$$

Besides, $B$ factors through a completely continuous linear operator due to the Dunford-Pettis property of the space $\ell^{1}$ whenever one of the aboves holds.

Proof. i) $\Rightarrow$ ii) Since the map $B$ is $\odot$-factorable, it factors through the linear map $T: \ell^{1} \rightarrow H$. Since $\mathcal{L}\left(\ell^{1}, H\right)=\Pi_{1}\left(\ell^{1}, H\right)$ by a result of the Grothendieck's Theorem, 
we obtain $T$ is a 1-summing operator and thus, $B$ satisfies the inequality given in statement (ii).

ii) $\Rightarrow$ iii) The integral domination given in the third statement is clearly obtained by Pietsch Domination Theorem (see 9, Theorem 2.12]).

iii) $\Rightarrow$ i) If the map $B$ has the integral domination then it is seen that $B\left(x_{1}, \ldots, x_{n}\right)$ $=0$ whenever $x_{k} \odot x_{l}=0$ for some different $k, l \in\{1, \ldots, n\}$. Thus, $B$ is zero $\odot-$ preserving and it is $\odot$-factorable by the main theorem of the paper.

We obtain a weaker result by considering some cotype-related properties. It is known that cotype 2 for a Banach space implies the Orlicz property (see 8 , Section 8.9]). Assume that $Y$ has Orlicz property and let $\sum_{i=1}^{n} \frac{1}{p_{i}}=1$ for $1 \leq p_{i}<\infty$ for $i=1, \ldots, n$. The following domination inequality holds for an $n$-linear $\odot$-factorable map $B: \times_{i=1}^{n} \ell^{p_{i}} \rightarrow Y$

$$
\left(\sum_{j=1}^{m}\left\|B\left(x_{j}^{1}, \ldots, x_{j}^{n}\right)\right\|_{Y}^{2}\right)^{1 / 2} \leq \sup _{\varepsilon_{j}=\{-1,1\}}\left\|\sum_{j=1}^{m} \varepsilon_{j} x_{j}^{1} \odot \ldots \odot x_{j}^{n}\right\|
$$

for all finite sets $\left\{x_{1}^{i}, \ldots, x_{m}^{i}\right\} \subset \ell^{p_{i}}(i=1, \ldots, n)$.

Lastly, we will give some results for $\odot$-factorable maps that are $\ell^{p}$-space valued. We will use Littlewood inequality that states $\mathcal{L}\left(\ell^{1}, \ell^{4 / 3}\right)=\Pi_{4 / 3,1}\left(\ell^{1}, \ell^{4 / 3}\right)$ (see 8 . Section 34.12]): if $B$ is defined on $\times_{i=1}^{n} \ell^{p_{i}}$ to $\ell^{4 / 3}$, then

$$
\left(\sum_{j=1}^{m}\left\|B\left(x_{j}^{1}, \ldots, x_{j}^{n}\right)\right\|_{4 / 3}^{4 / 3}\right)^{3 / 4} \leq k \sup _{z^{\prime} \in B_{\ell} \infty} \sum_{j=1}^{m}\left|\left\langle x_{j}^{1} \odot \ldots \odot x_{j}^{n}, z^{\prime}\right\rangle\right|
$$

for all finite sets $\left\{x_{j}^{i}\right\}_{j=1}^{m} \subset \ell^{p_{i}}(i=1, \ldots, n)$.

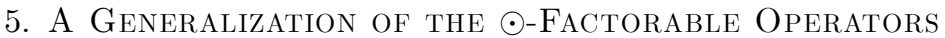

Let $\sum_{i=1}^{n} \frac{1}{p_{i}}=\frac{1}{r}$ for $1 \leq r, p_{i}<\infty(i=1, \ldots, n)$. Consider $n$ Banach spaces $X_{i}(i=1, \ldots, n)$ that are isomorphic to $\ell^{p_{i}}$ by the isomorphisms $P_{i}: X_{i} \rightarrow \ell^{p_{i}}$. Let us define the product $\odot_{\times_{i=1}^{n} P_{i}}: \times_{i=1}^{n} X_{i} \rightarrow \ell^{r}$ by

$$
\odot_{\times_{i=1}^{n} P_{i}}\left(f_{1}, \ldots, f_{n}\right)=P_{1}\left(f_{1}\right) \odot \ldots \odot P_{n}\left(f_{n}\right), \quad f_{i} \in X_{i} .
$$

This product can be illustrated by the following diagram;

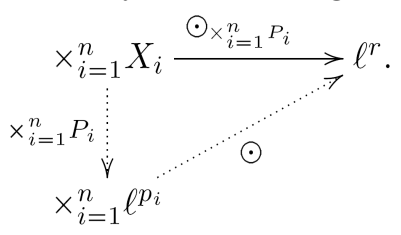

We will call a multilinear map $B: \times_{i=1}^{n} X_{i} \rightarrow Y$ is zero $\odot_{\times_{i=1}^{n} P_{i}}$-preserving if $B\left(f_{1}, \ldots, f_{n}\right)=0$ whenever $\odot_{P_{k} \times P_{l}}\left(f_{k}, f_{l}\right)=P_{k}\left(f_{k}\right) \odot P_{l}\left(f_{l}\right)=0$ for some $k, l \in$ $\{1, \ldots, n\}$ such that $k \neq l$. 
Theorem 9. Let $\sum_{i=1}^{n} \frac{1}{p_{i}}=\frac{1}{r}$ for $1 \leq r, p_{i}<\infty(i=1, \ldots, n)$. Consider the Banach spaces $X_{i}(i=1, \ldots, n)$ that are isomorphic to $\ell^{p_{i}}$ by means of the isomorphisms $P_{i}: X_{i} \rightarrow \ell^{p_{i}}$. For an n-linear map $B: \times_{i=1}^{n} X_{i} \rightarrow Y$, the following statements are equivalent.

(1) The operator $B$ is zero $\odot_{\times_{i=1}^{n} P_{i}}$-preserving.

(2) The map $B$ is $\odot_{\times_{i=1}^{n} P_{i}}$-factorable. That is, there is a linear operator $T$ : $\ell^{r} \rightarrow Y$ such that $B:=T \circ \odot_{\times_{i=1}^{n} P_{i}}$.

(3) There exists a $K>0$ such that the inequality below holds for every finite sets $\left\{f_{1}^{i}, \ldots, f_{m}^{i}\right\} \subset X_{i}(i=1, . ., n)$;

$$
\left\|\sum_{j=1}^{m} B\left(f_{j}^{1}, \ldots, f_{j}^{n}\right)\right\|_{Y} \leq K\left\|\sum_{j=1}^{m} P_{1}\left(f_{j}^{1}\right) \odot \ldots \odot P_{n}\left(f_{j}^{n}\right)\right\|_{r} .
$$

If one of the aboves is satisfied, then $B$ admits the following factorization;

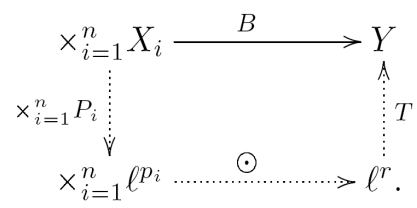

Proof. (1) $\Rightarrow$ (2) Let us assume that $B$ is zero $\odot_{\times_{i=1}^{n} P_{i}}$-preserving and define the map $\bar{B}=B \circ \times_{i=1}^{n} P_{i}^{-1}: \times_{i=1}^{n} \ell^{p_{i}} \rightarrow Y$. For the sequences $x_{i} \in \ell^{p_{i}}(i \in\{1, \ldots, n\})$, it is seen that $\bar{B}\left(x_{1}, . ., x_{n}\right)=B \circ \times_{i=1}^{n} P_{i}^{-1}\left(P_{1}\left(f_{1}\right), \ldots, P_{n}\left(f_{n}\right)\right)$, where $P_{i}\left(f_{i}\right)=x_{i}$ for $f_{i} \in X_{i}$. Since $B$ is zero $\odot_{\times_{i=1}^{n} P_{i}}$-preserving, it is obtained that $\bar{B}\left(x_{1}, . ., x_{n}\right)=$ $B\left(f_{1}, \ldots, f_{n}\right)=0$ whenever $x_{k} \odot x_{l}=P_{k}\left(f_{k}\right) \odot P_{l}\left(f_{l}\right)=0$ for some $k, l \in\{1, \ldots, n\}$. This shows zero $\odot$-preservation of the map $\bar{B}$ and therefore $\bar{B}$ is $\odot$-factorable by Theorem 3 . So we have that there is a linear operator $T: \ell^{r} \rightarrow Y$ such that $\bar{B}=T \circ \odot$. By the definition of $\bar{B}$, we obtain $B=\bar{B} \circ\left(\times_{i=1}^{n} P_{i}\right)=T \circ \odot \circ\left(\times_{i=1}^{n} P_{i}\right)=$ $T \circ \odot_{\times_{i=1}^{n} P_{i}}$, the desired factorization.

$(2) \Rightarrow(3)$ If the map $B$ is $\odot_{\times_{i=1}^{n} P_{i}}$ factorable then the map $\bar{B}=B \circ \times_{i=1}^{n} P_{i}^{-1}$ : $\times_{i=1}^{n} \ell^{p_{i}} \rightarrow Y$ is $\odot$-factorable. Indeed, for the $\odot \times_{i=1}^{n} P_{i}-$ factorable map $B$, there is a linear operator $T: \ell^{r} \rightarrow Y$ such that $B:=T \circ \odot_{\times_{i=1}^{n} P_{i}}$. Thus, $\bar{B}=T \circ \odot_{\times_{i=1}^{n} P_{i}} \circ$ $\times_{i=1}^{n} P_{i}^{-1}$. For the elements $f_{i} \in X_{i}$ that are $P_{i}\left(f_{i}\right)=x_{i} \in \ell^{p_{i}}$, we get

$$
\begin{aligned}
\bar{B}\left(x_{1}, \ldots, x_{n}\right) & =T \circ \odot_{\times_{i=1}^{n} P_{i}} \circ \times_{i=1}^{n} P_{i}^{-1}\left(x_{1}, \ldots, x_{n}\right) \\
& =T \circ \odot \times_{i=1}^{n} P_{i}\left(P_{1}^{-1}\left(x_{1}\right), \ldots, P_{n}^{-1}\left(x_{n}\right)\right) \\
& =T\left(P_{1} P_{1}^{-1}\left(x_{1}\right) \odot \ldots \odot P_{n} P_{n}^{-1}\left(x_{n}\right)\right) \\
& =T\left(x_{1} \odot \ldots \odot x_{n}\right) .
\end{aligned}
$$

This shows, $\bar{B}$ is $\odot$-factorable. By Lemma 2.3 given in 12 and Theorem 3 , the inequality given in the statement (3) is obtained. 
$(3) \Rightarrow(1)$ It is clear that $B$ is zero $\odot_{\times_{i=1}^{n}} P_{i}$-preserving under the assumption of the statement (3).

\section{Application: Representation As n-homogeneous Polynomial}

Recall that an $n$-linear map $B: \times{ }^{n} X \rightarrow Y$ is called symmetric if

$$
B\left(x_{1}, \ldots, x_{n}\right)=B\left(x_{\sigma(1)}, \ldots, x_{\sigma(n)}\right) \quad\left(x_{1}, \ldots, x_{n} \in X\right)
$$

for any permutation $\sigma$ of the first $n$ natural numbers. $\mathcal{L}_{s}^{n}\left(\times^{n} X, Y\right)$ denotes the space of symmetric multilinear operators defined on $X$ to $Y$.

Remark 10. Let $p \geq n$. It is easily seen that any $\odot$-factorable $n$-linear map $B: \times{ }^{n} \ell^{p} \rightarrow Y$ is symmetric. Indeed, the map $B$ factors through the linear map $T: \ell^{p / n} \rightarrow Y$ and thus

$$
B\left(x_{1}, \ldots, x_{n}\right)=T\left(x_{1} \odot \ldots \odot x_{n}\right)=T\left(x_{\sigma(1)} \odot \ldots \odot x_{\sigma(n)}\right)=B\left(x_{\sigma(1)}, \ldots, x_{\sigma(n)}\right)
$$

for all $x_{1}, \ldots, x_{n} \in \ell^{p}$ by the commutativity of the product $\odot$.

In addition to this, a symmetry is obtained for the general version. Let $X$ be isomorphic to the space $\ell^{p}$ by the isomorphism $P: X \rightarrow \ell^{p}$. Then any $\odot_{x^{n}}{ }-$ factorable $n$-linear map $B: \times^{n} X \rightarrow Y$ is symmetric.

Therefore, the following inclusions hold;

- $\mathcal{L}_{0}^{n}\left(\times^{n} \ell^{p}, Y\right) \subseteq \mathcal{L}_{s}^{n}\left(\times^{n} \ell^{p}, Y\right)$,

- $\mathcal{L}_{0}^{n}\left(\times^{n} X, Y\right) \subseteq \mathcal{L}_{s}^{n}\left(\times^{n} X, Y\right)$ if $X \cong \ell^{p}$.

We will give a counterexample to show that the symmetry does not imply zero $\odot$-preservation. Consider a bilinear map $B: \ell^{p} \times \ell^{p} \rightarrow \mathbb{R}$ defined by $B\left(x_{1}, x_{2}\right)=$ $\sum_{k=1}^{5} x^{1}(k) \cdot x^{2}(k)$. It is seen that $B$ is symmetric. For the sequences $x_{1}=$ $(1,1,0,1,0,-1,-1,0,0, \ldots)$ and $x_{2}=(1,1,1,0,1,1,1,0,0, \ldots)$ in $\ell^{p}$, it is obtained that $x_{1} \odot x_{2}=0$ but $B\left(x_{1}, x_{2}\right)=2$, thus $B$ is not zero $\odot$-preserving.

A map $P: X \rightarrow Y$ is called $n$-homogeneous polynomial if it is associated with an $n$-linear symmetric map $B: \times^{n} X \rightarrow Y$ such that $P(x)=B(x, \ldots, x)$ for all $x \in X$. The class of $n$-homogeneous polynomials is a Banach space under the norm $\|P\|=\sup _{\|x\|=1}\|P(x)\|$. It will be denoted by $\mathcal{P}\left({ }^{n} X, Y\right)$. We refer the book 10 for more information about polynomials.

An $n$-homogeneous polynomial defined on the Banach algebra $X$ is called orthogonally additive if $P(x+y)=P(x)+P(y)$ whenever $x y=0$ for $x, y \in X$. Similarly we will call an $n$-homogeneous polynomial defined on the Banach space $X$ orthogonally additive if $P(x+y)=P(x)+P(y)$ whenever $x \circledast y=0$ for $x, y \in X$ and an n.p. product $\circledast$. We denote by $\mathcal{P}_{0}\left({ }^{n} X, Y\right)$ the space of $n$-homogeneous orthogonally additive polynomials from $X$ to $Y$. We will write $\mathcal{P}_{0}\left({ }^{n} X\right)$ for $Y=\mathbb{R}$.

The Banach space of $n$-homogeneous orthogonally additive polynomials is closely related to the zero product preserving $n$-linear operators and several papers can be found in this direction in the literature (see $[3,5,12,15,16$, and references therein). Now we will give a generalization of the isomorphisms between orthogonally additive $n$-homogeneous polynomial forms and sequences given in the papers 15$]$ and 16 . 
Theorem 11. Let $1 \leq n \leq p<\infty$. There is an onto isometry between the spaces $\mathcal{L}\left(\ell^{p / n}, Y\right)$ and $\mathcal{P}_{0}\left({ }^{n} \ell^{p}, Y\right)$. Particularly, $\mathcal{P}_{0}\left({ }^{n} \ell^{p}\right)=\left(\ell^{p / n}\right)^{*}$ for a scalar field range.

Proof. Consider a linear continuous operator $T \in \mathcal{L}\left(\ell^{p / n}, Y\right)$. It is seen that $T$ gives a $\odot$-factorable $n$-linear map $B_{T}: \times{ }^{n} \ell^{p} \rightarrow Y$ defined by $T(x)=T\left(x_{1} \odot \ldots \odot x_{n}\right)=$ $B\left(x_{1}, \ldots, x_{n}\right)$ for all $x_{i} \in \ell^{p}(i=1, \ldots, n)$ such that $x_{1} \odot \ldots \odot x_{n}=x \in \ell^{p / n}$. Due to the symmetry of the $\odot$-factorable map $B_{T}$, an $n$-homogeneous polynomial $P_{B_{T}}: \ell^{p} \rightarrow Y$ is obtained such that it is orthogonally additive. Indeed, for all $x, y \in \ell^{p}$

$$
\begin{aligned}
P_{B_{T}}(x+y) & =B_{T}(x+y, \ldots, x+y) \\
& =\sum_{k=0}^{n}\left(\begin{array}{l}
n \\
k
\end{array}\right) B_{T}(x, \ldots, x, y, \stackrel{n-k}{\cdots}, y) \\
& =B_{T}(x, \stackrel{n}{n}, x)+B_{T}(y, \cdots, y) \\
& =P_{B_{T}}(x)+P_{B_{T}}(y) .
\end{aligned}
$$

whenever $x \odot y=0$, thus $P_{B_{T}}$ is orthogonally additive. Thus the linear correspondence $T \rightarrow P_{B_{T}}$ defines an orthogonally additive $n$-homogeneous polynomial $P_{B_{T}}$ for every $T$ by $T\left(x^{n}\right)=P_{B_{T}}(x)$, where $x^{n}=x \odot \stackrel{n}{ } \odot x$. Let us show the isometry now.

$$
\|T\|=\sup _{\|x\|_{p / n} \leq 1}\|T x\|=\sup _{\left\|x^{1 / n}\right\|_{p} \leq 1}\left\|P\left(x^{1 / n}\right)\right\|=\sup _{\|y\|_{p} \leq 1}\|P(y)\|=\|P\| .
$$

For the surjectivity, let us consider an orthogonally additive $n$-homogeneous polynomial $P \in \mathcal{P}_{0}\left({ }^{n} \ell^{p}, Y\right)$. This polynomial defines a 1-homogeneous map $T$ by $T(x)=$ $P\left(x^{1 / n}\right)$ for all $x=\{x(k)\}_{k=1}^{\infty} \in \ell^{p / n}$ where $x^{1 / n}=\left\{|x(k)|^{1 / n} \operatorname{sign}(x(k))\right\}_{k=1}^{\infty}$ such that $x=\left\{|x(k)|^{1 / n} \operatorname{sign}(x(k)) \cdot n \cdot \cdot|x(k)|^{1 / n} \operatorname{sign}(x(k))\right\}_{k=1}^{\infty}=x^{1 / n} \odot n \cdot \odot x^{1 / n} \in \ell^{p / n}$. The map $T$ is linear. Indeed, to see this consider the sequences $x_{1}^{\prime}=\sum_{k=1}^{m} x^{1}(k)$. $\chi_{\{k\}}$ and $x_{2}^{\prime}=\sum_{k=1}^{m} x^{2}(k) \cdot \chi_{\{k\}}$ defined by the sequences $x_{1}, x_{2} \in \ell^{p / n}$.

Since $\left(x_{1}^{\prime}+x_{2}^{\prime}\right)^{1 / n}=\sum_{k=1}^{m}\left(x^{1}(k)+x^{2}(k)\right)^{1 / n} \cdot \chi_{\{k\}}$, by using the $n$-homogenity and orthogonally additivity of the polynomial $P$, we get that

$$
\begin{aligned}
T\left(x_{1}^{\prime}+x_{2}^{\prime}\right) & =P\left(\left(x_{1}^{\prime}+x_{2}^{\prime}\right)^{1 / n}\right)=P\left(\sum_{k=1}^{m}\left(x^{1}(k)+x^{2}(k)\right)^{1 / n} \cdot \chi_{\{k\}}\right) \\
& =\sum_{k=1}^{m} P\left(\left(x^{1}(k)+x^{2}(k)\right)^{1 / n} \cdot \chi_{\{k\}}\right)=\sum_{k=1}^{m}\left(x^{1}(k)+x^{2}(k)\right) P\left(\chi_{\{k\}}\right) \\
& =P\left(\sum_{k=1}^{m}\left(x^{1}(k)\right)^{1 / n} \cdot \chi_{\{k\}}\right)+P\left(\sum_{k=1}^{m}\left(x^{2}(k)\right)^{1 / n} \cdot \chi_{\{k\}}\right) \\
& =P\left(\left(x_{1}^{\prime}\right)^{1 / n}\right)+P\left(\left(x_{2}^{\prime}\right)^{1 / n}\right)=T\left(x_{1}^{\prime}\right)+T\left(x_{2}^{\prime}\right) .
\end{aligned}
$$


Since $x_{1}=\lim _{m \rightarrow \infty} \sum_{k=1}^{m} x^{1}(k) \cdot \chi_{\{k\}}$ and $x_{2}=\lim _{m \rightarrow \infty} \sum_{k=1}^{m} x^{2}(k) \cdot \chi_{\{k\}}$, it is obtained that

$$
\begin{aligned}
T\left(x_{1}+x_{2}\right) & =T\left(\lim _{m \rightarrow \infty} x_{1}^{\prime}+\lim _{m \rightarrow \infty} x_{2}^{\prime}\right) \\
& =\lim _{m \rightarrow \infty} T\left(x_{1}^{\prime}+x_{2}^{\prime}\right)=\lim _{m \rightarrow \infty}\left(T\left(x_{1}^{\prime}\right)+T\left(x_{2}^{\prime}\right)\right) \\
& =T\left(x_{1}+x_{2}\right) .
\end{aligned}
$$

Thus, every orthogonally additive $n$-homogeneous polynomial $P$ defines a linear map $T \in \mathcal{L}\left(\ell^{p / n}, Y\right)$. We can illustrate this isometry by the following diagram;

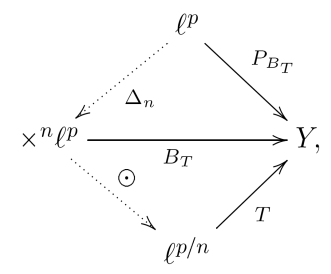

where $\Delta_{n}$ is the canonical embedding called diagonal mapping from $\ell^{p}$ to $\times^{n} \ell^{p}$ used to define the $n$-homogeneous polynomials.

Particularly, every $n$-homogeneous polynomial form $P$ in $\mathcal{P}_{0}\left({ }^{n} \ell^{p}\right)$ is represented by a sequence in the space $\ell^{p /(p-n)}$.

Corollary 12. $\mathcal{L}\left(\ell^{1}, Y\right)=\mathcal{P}_{0}\left({ }^{n} \ell^{n}, Y\right)$ and every orthogonally additive $n$-homogenous polynomial $P: \ell^{n} \rightarrow \mathbb{R}$ is represented by a bounded scalar valued sequence.

From Corollary 5 , Theorem 11 and Corollary 12 , we get the following isometries;

$\star \mathcal{L}_{0}^{n}\left(\times^{n} \ell^{p}, Y\right)=\mathcal{P}_{0}\left({ }^{n} \ell^{p}, Y\right)$, where $p \geq n$.

$\star \mathcal{L}_{0}^{n}\left(\times^{n} \ell^{n}, Y\right)=\mathcal{P}_{0}\left({ }^{n} \ell^{n}, Y\right)$

$\star \mathcal{L}_{0}^{n}\left(\times^{n} \ell^{p}\right)=\mathcal{P}_{0}\left({ }^{n} \ell^{p}\right)$

$\star \mathcal{L}_{0}^{n}\left(\times{ }^{n} \ell^{n}\right)=\mathcal{P}_{0}\left({ }^{n} \ell^{n}\right)$.

We can give some isomorphisms for the $\odot_{x^{n} P}$-factorable maps as follows;

Corollary 13. Let $1 \leq n \leq p<\infty$ and $P: E \rightarrow \ell^{p}$ is an isomorphism. There is an isomorphism between the spaces $\mathcal{L}\left(\ell^{p / n}, Y\right)$ and $\mathcal{P}_{0}\left({ }^{n} E, Y\right)$. Particularly, $\mathcal{P}_{0}\left({ }^{n} E\right)=$ $\left(\ell^{p / n}\right)^{*}$ for a scalar field range.

Let us finish the paper with an example.

Example 14. Let $\sum_{i=1}^{n} \frac{1}{p_{i}}=\frac{1}{r}$ for $1 \leq r, p_{i}<\infty$ for $i=1, \ldots, n$. Recall that a multilinear form $B: \times_{i=1}^{n} \ell^{p_{i}} \rightarrow \mathbb{C}$ defined by $B\left(x_{1}, \ldots, x_{n}\right)=\sum_{k=1}^{\infty} \alpha_{k} \cdot x_{k}^{1} \cdot \ldots \cdot x_{k}^{n}$ is called diagonal operator, where $\left\{\alpha_{k}\right\}$ is a bounded sequence. Clearly, it is seen by the definition that $B\left(x_{1}, \ldots, x_{n}\right)=0$ whenever $x_{k} \odot x_{l}=0$ for some $k, l \in\{1,2, \ldots, n\}$. Therefore, it is zero product preserving and there is a linear form $T: \ell^{r} \rightarrow \mathbb{C}$ such that $B\left(x_{1}, \ldots, x_{n}\right)=T(x)$, where $x_{1} \odot \ldots . \odot x_{n}=x$. Besides, if we consider $p_{i}=$ $\ldots=p_{n}=p$, then we obtain that the zero product preserving map $B: \times^{n} \ell^{p} \rightarrow \mathbb{C}$ 
has a factorization through the linear form $T: \ell^{p / n} \rightarrow \mathbb{C}$. Since this gives the symmetry of the form $B: \times^{n} \ell^{p} \rightarrow \mathbb{C}$, we get the diagonal map $B$ is associated with an orthogonally additive $n$-homogeneous diagonal polynomial form $P: \ell^{p} \rightarrow \mathbb{C}$.

\section{REFERENCES}

[1] Alaminos, J., Brešar, M., Extremera, J., Villena, A. R., Maps preserving zero products, Studia Math., 193 (2) (2009), 131-159.

[2] Alaminos, J., Brešar, M., Spenko, S., Villena A. R., Orthogonally additive polynomials and orthosymmetric maps in Banach algebras with properties $\mathbb{A}$ and $\mathbb{B}$, Proceedings of the Edinburgh Mathematical Society, 59 (3) (2016), 559-568.

[3] Alaminos, J., Extremera, J., Godoy, M. L. C., Villena, A. R., Orthogonally additive polynomials on convolution algebras associated with a compact group, J Math Anal Appl., 472 (1) (2019),285-302.

[4] Ben Amor, F., On orthosymmetric bilinear maps, Positivity, 14 (2010), 123-134.

[5] Benyamini, Y., Lassalle, S., Llavona, J. G., Homogeneous orthogonally additive polynomials on Banach lattices, Bull Lond Math Soc., 383 (2006), 459-469.

[6] Boulabiar, K., Buskes, G., Vector lattice powers: f-algebras and functional calculus, Comm Algebra., 344 (2006), 1435-1442.

[7] Bu, Q., Buskes, G., Kusraev, A. G., Bilinear Maps on Products of Vector Lattices: A Survey, In: Boulabiar K., Buskes G., Triki A. (eds) Positivity. Trends in Mathematics, (2007), 97126.

[8] Defant, A., Floret, K., Tensor norms and operator ideals, North-Holland Math. Stud. Vol. 176, North-Holland, Amsterdam, Elsevier, 1993.

[9] Diestel, J., Jarchow H., Tonge, A., Absolutely Summing Operators, Vol. 43. Cambridge University Press, 1995.

[10] Dineen, S., Complex Analysis on Infinite Dimensional Spaces, Springer-Verlag, London, 1999.

[11] Erdoğan, E., Calabuig, J. M., Sánchez Pérez, E. A., Convolution-continuous bilinear operators acting in Hilbert spaces of integrable functions, Ann. Funct. Anal., 9 (2) (2018), 166-179.

[12] Erdoğan, E., Factorization of multilinear operators defined on products of function spaces, Linear and Multilinear Algebra, (2020), https://dx.doi.org/10.1080/03081087.2020.1715334

[13] Erdoğan, E., Gök, Ö., Convolution Factorability of Bilinear Maps and Integral Representations, Indag. Math., 29 (5) (2018), 1334-1349.

[14] Erdoğan, E., Sánchez Pérez, E. A., Gök, Ö., Product factorability of integral bilinear operators on Banach function spaces, Positivity, 23 (3) (2019), 671-696.

[15] Ibort, A., Linares, P., Llavona, J.G., A representation theorem for orthogonally additive polynomials on Riesz spaces, Rev Mat Complut., 251 (2012), 21-30.

[16] Sundaresan, K., Geometry of spaces of polynomials on Banach lattices in: Applied Geometry and Discrete Mathematics, DIMACS Ser. Discrete Math. Theoret. Comput. Sci., Amer. Math. Soc., Providence RI, vol 4 (1991), pp 571-586. 